\title{
Impaired glutamate homeostasis in the nucleus accumbens in human cocaine addiction
}

Engeli, Etna J E ; Zoelch, Niklaus ; Hock, Andreas ; Nordt, Carlos ; Hulka, Lea M ; Kirschner, Matthias ; Scheidegger, Milan ; Esposito, Fabrizio ; Baumgartner, Markus R ; Henning, Anke ; Seifritz, Erich ; Quednow, Boris B ; Herdener, Marcus

\begin{abstract}
Cocaine addiction is characterized by overwhelming craving for the substance, which drives its escalating use despite adverse consequences. Animal models suggest a disrupted glutamate homeostasis in the nucleus accumbens to underlie addiction-like behaviour. After chronic administration of cocaine, rodents show decreased levels of accumbal glutamate, whereas drug-seeking reinstatement is associated with enhanced glutamatergic transmission. However, due to technical obstacles, the role of disturbed glutamate homeostasis for cocaine addiction in humans remains only partially understood, and accordingly, no approved pharmacotherapy exists. Here, we applied a tailored proton magnetic resonance spectroscopy protocol that allows glutamate quantification within the human nucleus accumbens. We found significantly reduced basal glutamate concentrations in the nucleus accumbens in cocaine-addicted $(\mathrm{N}=26)$ compared to healthy individuals $(\mathrm{N}=30)$, and increased glutamate levels during cue-induced craving in cocaine-addicted individuals compared to baseline. These glutamatergic alterations, however, could not be significantly modulated by a short-term challenge of $\mathrm{N}$-acetylcysteine (2400 mg/day on two days). Taken together, our findings reveal a disturbed accumbal glutamate homeostasis as a key neurometabolic feature of cocaine addiction also in humans. Therefore, we suggest the glutamatergic system as a promising target for the development of novel pharmacotherapies, and in addition, as a potential biomarker for a personalised medicine approach in addiction.
\end{abstract}

DOI: https://doi.org/10.1038/s41380-020-0828-z

Posted at the Zurich Open Repository and Archive, University of Zurich

ZORA URL: https://doi.org/10.5167/uzh-188394

Journal Article

Accepted Version

Originally published at:

Engeli, Etna J E; Zoelch, Niklaus; Hock, Andreas; Nordt, Carlos; Hulka, Lea M; Kirschner, Matthias; Scheidegger, Milan; Esposito, Fabrizio; Baumgartner, Markus R; Henning, Anke; Seifritz, Erich; Quednow, Boris B; Herdener, Marcus (2021). Impaired glutamate homeostasis in the nucleus accumbens in human cocaine addiction. Molecular Psychiatry, 26(9):5277-5285.

DOI: https://doi.org/10.1038/s41380-020-0828-z 


\section{Impaired glutamate homeostasis in the nucleus accumbens in human cocaine addiction}

Etna J. E. Engeli, MSc1*, Niklaus Zoelch, PhD2,3,4, Andreas Hock, PhD 2,4, Carlos Nordt, PhD1, Lea M. Hulka, PhD 1, Matthias Kirschner MD1,5, Milan Scheidegger, MD, PhD 4, Fabrizio Esposito, PhD6, Markus R. Baumgartner, PhD7, Anke Henning, $\mathrm{PhD} 2,8,9,10$, Erich Seifritz MD4,8,11, Boris B. Quednow PhD8,11,12, Marcus Herdener, MD1

${ }_{1}$ Centre for Addictive Disorders, Department of Psychiatry, Psychotherapy and Psychosomatics, Psychiatric Hospital, University of Zurich, Switzerland.

2 Institute for Biomedical Engineering, University of Zurich and Swiss Federal Institute of Technology Zurich, Switzerland.

3 Institute of Forensic Medicine, University of Zurich, Switzerland.

4 Department of Psychiatry, Psychotherapy and Psychosomatics, Psychiatric Hospital, University of Zurich, Switzerland.

5 Montreal Neurological Institute, McGill University, Montreal, Quebec, Canada.

6 Department of Medicine, Surgery and Dentistry, Scuola Medica Salernitana, University of Salerno, Baronissi, Salerno, Italy.

7 Centre for Forensic Hair Analytics, Zurich Institute of Forensic Medicine, University of Zurich, Switzerland.

8 Zurich Centre for Integrative Human Physiology, University of Zurich, Switzerland.

9 Max-Planck-Institute for Biological Cybernetics, Tuebingen, Germany.

10 Institute of Physics, Ernst-Moritz-Arndt University Greifswald, Germany.

11 Neuroscience Centre Zurich, University of Zurich and Swiss Federal Institute of Technology Zurich, Switzerland.

12 Experimental and Clinical Pharmacopsychology, Department of Psychiatry, Psychotherapy and Psychosomatics, Psychiatric Hospital, University of Zurich, Switzerland.

* Corresponding author:

Etna J. E. Engeli, MSc

Centre for Addictive Disorders

Department of Psychiatry, Psychotherapy and Psychosomatics,

Psychiatric Hospital, University of Zurich

Selnaustrasse 9, CH-8001 Zurich, Switzerland

Tel.: +41 (0)44205 5800

E-Mail: etna.engeli@bli.uzh.ch 
Cocaine addiction is characterized by overwhelming craving for the substance, which drives its escalating use despite adverse consequences. Animal models suggest a disrupted glutamate homeostasis in the nucleus accumbens to underlie addiction-like behaviour. After chronic administration of cocaine, rodents show decreased levels of accumbal glutamate, whereas drug-seeking reinstatement is associated with enhanced glutamatergic transmission. However, due to technical obstacles, the role of disturbed glutamate homeostasis for cocaine addiction in humans remains only partially understood, and accordingly, no approved pharmacotherapy exists. Here, we applied a tailored proton magnetic resonance spectroscopy protocol that allows glutamate quantification within the human nucleus accumbens. We found significantly reduced basal glutamate concentrations in the nucleus accumbens in cocaine-addicted $(\mathrm{N}=26)$ compared to healthy individuals $(\mathrm{N}=30)$, and increased glutamate levels during cue-induced craving in cocaine-addicted individuals compared to baseline. These glutamatergic alterations, however, could not be significantly modulated by a shortterm challenge of $\mathrm{N}$-acetylcysteine ( $2400 \mathrm{mg} /$ day on two days). Taken together, our findings reveal a disturbed accumbal glutamate homeostasis as a key neurometabolic feature of cocaine addiction also in humans. Therefore, we suggest the glutamatergic system as a promising target for the development of novel pharmacotherapies, and in addition, as a potential biomarker for a personalised medicine approach in addiction. 
Structural and functional adaptations within the neural reward circuitry are at the core of cocaine addiction (CA). These alterations promote craving for cocaine, while striving for naturally rewarding experiences is blunted 1-4. This dysfunctional reward motivation provokes frequent substance taking, and thus can create a vicious circle of addictive behaviours. The vulnerability for relapse to substance use is linked to multiple changes in reward-relevant neurotransmitter systems, particularly within the nucleus accumbens (Nacc) that serves as an integrative hub for translating value representation into action initiation5. The current understanding of these alterations underlying addictive behaviours has been decisively shaped by insights from animal models. In particular, rodent studies of CA identified molecular mechanisms behind drug-seeking behaviour. They indicate destabilising consequences of chronic substance administration on glutamate (Glu) homeostasis, especially on the balance between synaptic and non-synaptic Glu in the Nacc6,7. Usually, Glu signalling is dynamically regulated by astroglial cells which govern its release and uptakes. However, these glial regulatory activities are dysfunctional after chronic cocaine administration 8 , leading to decreased basal Glu concentrations in the extrasynaptic space of the Nacc core during phases of abstinence. In turn, relapse-provoking exposure to cocaine challenges or conditioned cues result in enhanced stimulation of Glu-ergic transmission9. Given that the glial regulation is disrupted, the concentration of synaptically released Glu rises in the Nacc core6. Furthermore, this increase in extrasynaptic Glu is associated with amplified substanceseeking 10.

Gaining a better understanding of the neurobiological adaptations underlying maladaptive substance-seeking in humans is necessary for the targeted development of pharmacotherapies for CA, which are lacking so far11. Despite the animal model-based understanding of substance-related adaptations within the Glu system in the Nacc, sparse evidence exists for the translational validity of these models into humans. Extensive efforts have been made to capture accumbal Glu homeostasis by applying proton magnetic resonance spectroscopy $(1 \mathrm{H}$ MRS) as a non-invasive technique to determine concentrations of target metabolites such as Glu in the brain. However, due to technical challenges of $1 \mathrm{H}-\mathrm{MRS}$ application, specific evaluation of Glu homeostasis within the Nacc of humans with CA has not been achieved yet12-15. Conventional $1 \mathrm{H}-\mathrm{MRS}$ measures yield rather low sensitivity, thus it has been challenging to assess the concentration of Glu in the human brain (around 6-12.5 mmol/L) that is $\sim 4000-7000$ times lower than the corresponding concentration of water 16 . To compensate for the low Glu concentration, larger 1H-MRS acquisition voxels are 
conventionally required to ensure sufficient signal-to-noise ratio (SNR). However, the size of the Nacc (1-2 $\left.\mathrm{cm}_{3}\right)$ requires a small ${ }_{1} \mathrm{H}-\mathrm{MRS}$ voxel to assess Glu specifically within this region17. Alternatively, to still enable region-specific measurements with sufficient SNR, many separate measurements can be assessed and averaged to compensate for the small voxel size. However, signal averaging requires a steady measurement environment over time. Unfortunately, vessels and ventricles surrounding the Nacc generate pulsating motion and magnetic susceptibility changes, which distort MRS line shape and limit the achievable SNR. To overcome the addressed issues, we used the water peak with its high SNR as a reference for averaging the signals by applying non-water suppressed metabolite cycling ${ }_{1} \mathrm{H}-\mathrm{MRS} 18-20$ to increase the SNR, decrease the linewidth, and thereby enable good spectral quality.

A further difficulty for neurometablic comparison between clinical and healthy populations in general is that metabolic concentrations have previously been evaluated as a relative measure only, defined as the ratio of the metabolite of interest to either creatine or water. Since these reference molecules could potentially be altered in patients, absolute quantification of metabolites of interest is necessary to unambiguously identify pathophysiological shifts of metabolites of interest. Therefore, we applied the principle of reciprocity21 to achieve an absolute quantification of metabolite concentrations instead of relative measures.

With this tailored $1 \mathrm{H}-\mathrm{MRS}$ we first aimed at quantifying accumbal Glu concentrations in individuals with cocaine addiction (CAI) and in healthy control individuals (HCI) during abstinence to test the glutamate homeostasis hypothesis of addiction in humans. Further, to address the potential role of Glu-ergic adaptations during cocaine craving in humans, Glu levels were additionally assessed when the desire to use cocaine was provoked with cocaine cues. Moreover, we investigated the restoring effect of a N-acetylcysteine challenge $(\mathrm{N}$ AC)22, that has shown to successfully counterbalance disruptions in Glu homeostasis in animal models of addiction23. We expected that CAI would show decreased Glu concentrations during abstinence and increased concentrations during craving. For N-AC, we hypothesised that it has the potential to normalise Glu and craving levels.

\section{Materials and Methods}

\section{Participants}

The study sample consisted of $26 \mathrm{CAI}$ and $30 \mathrm{HCI}$ who were recruited via online and in-house advertisement. Inclusion criteria for CAI were a minimum average cocaine use of $1 \mathrm{~g}$ per week 
within the last 6 months and a diagnosis of cocaine dependence according to the Diagnostic and Statistical Manual of Mental Disorders, Fourth Edition (DSM-IV)24. Exclusion criteria for CAI were other DSM-IV axis I psychiatric disorders including dependence of other psychoactive substances (excluding tobacco dependence and attention-deficit/hyperactivity disorder). Exclusion criteria of HCI were regular use of psychoactive substances with exception of current alcohol and irregular cannabis use. Additionally, also DSM-IV axis I psychiatric disorders (excluding tobacco dependence) lead to exclusion of HCI. General exclusion criteria for all participants were contraindication for magnetic resonance imaging, allergy from N-AC, severe somatic diseases, neurological disorders, prior head injury, pregnancy, lack of contraception, family history of severe DSM-IV axis I psychiatric disorders, and concurrent participation in another clinical trial.

All participants gave written informed consent in accordance with the declaration of Helsinki before study participation and received financial compensation after study completion or termination. The study was approved by the Cantonal Ethics Committee of Zurich (KEK ZH No.: 2014-0010).

\section{H-MRS data acquisition and analysis}

1H-MRS measurements were performed on a Philips Achieva 3T whole-body scanner equipped with a 32-channel receive-only phased-array head coil (Philips Healthcare, Best, The Netherlands). High-resolution anatomical images $(1 \times 1 \times 1 \mathrm{~mm} 3)$ were acquired using a standard T1-weighted 3D turbo field echo sequence. ${ }_{1} \mathrm{H}-\mathrm{MRS}$ spectra were obtained by means of non-water suppressed Point-RESolved Spectroscopy (PRESS) preceded by a metabolite-cycling pulse combined with inner-volume saturation (IVS)19. The PRESS localisation sequence was set to a repetition time of $2500 \mathrm{~ms}$, echo time of $32 \mathrm{~ms}$ and spectral bandwidth of $2000 \mathrm{~Hz}$. The metabolite-cycling technique with application of a shift-sensitive asymmetric adiabatic inversion pulse allowed alternately inverting either up- or downfield metabolites without affecting the water signal16,18-20. This enables simultaneous analyses of the water peak and metabolite signals after separating metabolite peaks from the water peak and sideband artefacts by either adding or subtracting consecutively acquired echoes. The unsuppressed water peak with its high SNR can be used as a reference for frequency alignment, phase and eddy current correction of all individual signals before averaging to enable optimal spectral quality. After metabolite-cycling pulses, IVS was implemented to enable precise voxel placement, to reduce chemical shift displacement errors between metabolites, and to reduce flow artefacts by applying six saturation bands (Fig. 1a; blue) 
based on highly selective, broadband radiofrequency pulses with polynomial phase response. Based on the T1-weighted image, the 1 H-MRS voxel (effective size after IVS: $9.4 \times 18.8 \times$ $8.4 \mathrm{~mm} 3$ ) was positioned and tilted to cover the left Nacc. In four blocks of 128 signals a total of 512 signals were achieved, which lasted 22.5 minutes.

For quantification of spectral data, LCModel 25 with a set of basis spectra, consisting of 18 metabolites, was used (Fig. 1b). To account for potential cerebrospinal fluid fraction in the measured voxel, the T1-weighted images were segmented using SPM826. Absolute quantification of metabolites was performed using an approach exploiting the phantom replacement method. To ensure adequate correction of differences between the acquisition of the phantom and the in-vivo measurement including corrections for coil loading and B1 inhomogeneity, the principle of reciprocity was applied as described in more details by Zoelch and colleagues21 and in the Supplementary Information. An in-house patch for the code of the Philips scanner, allowing for the use of the metabolite cycling technique and the absolute quantification method, was used and is available upon request.

\section{Craving paradigm}

During the ${ }_{1} \mathrm{H}-\mathrm{MRS}$ measurements, all participants watched two customised films, one as a control condition and the other one to provoke the desire to use cocaine. The first film showed video clips of a chess game to assess basal Glu levels (neutral film), while the second film showed clips of cocaine preparation and consumption to measure craving-induced Glu (cocaine film). The film order was not randomised to avoid carry-over effects of cocaine craving. These films were specifically created for this study in cooperation with professional actors and filmmakers to enable (a) accurate comparison between equivalent experimental and neutral condition; in both films, an interaction between two male protagonists is featured and both clips incorporate the same objects except for the chessboard and the cocaine paraphernalia, respectively. A customised cocaine film further allows (b) to expose participants to locally characteristic paraphernalia, and thereby the stimulus overcomes cultural diversity in substance use that might otherwise compromise authenticity. These authentic visual stimuli were accompanied by auditory stimuli such as the clicking sound of the chess pieces or the grinding and sniffing of cocaine to allow an immersive experience. In both films, the video clips were interleaved with blank black screens of 2.5 minutes. Before film presentations, participants were asked in a written instruction to empathise with the scenes shown in the video clips and to recollect similar autobiographic experiences during black pauses. 
Impaired glutamate homeostasis...

Subjective craving was assessed on a visual analogue scale ranging from 0 to 10 with intervals of $0.25(0=$ no craving; $10=$ strong craving $)$. Participants rated their craving on this scale with a computer mouse while lying in the scanner, directly before and after each film.

\section{Pharmacological challenge}

The pharmacological challenge comprised the cysteine prodrug $\mathrm{N}-\mathrm{AC}$ that is approved as a mucolytic and to treat acetaminophen overdose. A number of animal studies administering N$\mathrm{AC}$, which binds to cysteine-glutamate antiporters, were able to regulate Glu synthesis and cycling, and thus successfully modulate alterations in the Glu homeostasis after chronic cocaine administration.

The challenge was given as a dose of $2400 \mathrm{mg} /$ day on two consecutive days. The dosage was selected based on a study assessing the safety and tolerability of three doses, showing no significant difference between 1200, 2400, or $3600 \mathrm{mg} /$ day in terms of side effects 27. Additionally, preliminary data suggests that the dosage of $2400 \mathrm{mg} /$ day N-AC is potentially effective on craving, relapse, and on Glu levels in the cingulate gyrus in addiction 23. Here, the main goal was to investigate the potential impact of $\mathrm{N}-\mathrm{AC}$ on the Glu homeostasis in the Nacc. The treatment period of two days was therefore chosen to be short enough to maximize compliance and to minimize dropouts, yet long enough to see a potential effect according to previous reports 23 . The effect of N-AC was tested against a placebo, in the form of identical capsules of mannitol, to enable a randomized, double-blind, placebo-controlled and crossover procedure. The first dose of either N-AC or placebo was handed out to our participants on their assessment visit. The sequence of $\mathrm{N}$-AC and placebo sessions were randomized and counterbalanced to avoid order and sequence effects. Participants were instructed to take the first dose one day prior to the first measurement day, $1200 \mathrm{mg}$ in the morning and in the evening. On the measurement day itself, the second dose of $2400 \mathrm{mg}$ was administered on site one hour prior to the $1 \mathrm{H}-\mathrm{MRS}$ measurement, since peak plasma concentration of N-AC occurs $\sim 1-2$ hours after ingestion28. After the first measurement day, participants were crossed over to receive the other compound in the same administration regimen for the second measurement. Both measurement sessions were performed the same way. To ensure complete washout of the compounds, the sessions were separated by an interval of 14 days ( \pm 4 days). For more details, see Supplementary Information and Supplementary Fig. S1.

\section{Statistical analysis}


All analyses were performed in a sample consisting of $26 \mathrm{CAI}$ and $30 \mathrm{HCI}$, unless stated otherwise. For detailed description of data exclusion, see Supplementary Information. In order to allow the inclusion of all available data from every subject despite missing of single measurements, a multilevel approach was applied. Uneven data sets and irregular time intervals between measurement points were taken into account 28 , since random coefficients allow modelling the time course as continuous variable based on the assumption that slopes and intercepts vary randomly across individuals29. The random coefficient model estimated the impact of cocaine addiction (Table 1; CAI) and cocaine-cue stimulation (Table 1; cocaine film) as fixed effects on either the dependent variable Glu or craving to investigate their differences between groups and conditions (Figs. 2 and 3, respectively). The same model was estimated for Glx. We included time, age, sex, and duration of abstinence (analysis of urine samples and log-transformed hours since last cocaine use prior to measurement) as predicting variables in our multilevel models. None of these variables had an influence on Glu and craving levels before and after stimulation with the cocaine film, and were therefore omitted from the final models. All random coefficient models were fitted using SAS V9.4 with the function proc mixed following Brown and Prescott29 or proc nlmixed when accounting for variance inhomogeneity. Variance was observed to be inhomogeneous for craving as dependent variable. As the results did not differ substantially, only the model assuming variance homogeneity between groups is presented. Examination of possible outliers, test for normal distribution, and all other statistical calculations were performed with SPSS 25.0. Since guidelines regarding effect sizes for multilevel models have not yet been established, we calculated effect sizes using Cohen's $d$ for group differences of main interest taking into account their respective sample sizes.

\section{Results}

\section{Demographic characteristics and cocaine consumption}

The groups were matched regarding age, sex, education, verbal intelligence, and smoking status. In CAI, mean cocaine consumption rate and dose within the last six months was 3.40 times per week ( \pm 1.89 s.d., range: 0.46 - 7) and 5.52 grams per week ( \pm 10.03 s.d., range: 1 52.50). The main route of cocaine administration was intranasal (22 CAI), while 4 CAI were primarily inhaling cocaine. For full characterization of the final sample regarding demographics, matching and substance use, see Supplementary Table S1 and S2. 


\section{Craving induction}

Overall, CAI showed substantially higher craving ratings than $\mathrm{HCI}(P<0.0001, d=1.67$, $95 \%$ CI $[1.06,2.28], d$ calculated for pre neutral film HCI vs. CAI). Craving ratings increased strongly after presentation of the cocaine film only among CAI $(P=0.0002, d=0.85,95 \%$ CI $[0.28,1.42]$ ), while HCI did not report any cocaine craving (Fig. 2).

\section{H-MRS quality}

According to general MRS quality criteria from the MR Spectroscopy Consensus Group30, the upper limit for linewidth (full-width at half-maximum peak height) was defined as $<12.7$ $\mathrm{Hz}(0.1 \mathrm{ppm}) 30$. The threshold for sufficient SNR was set $>10$. Based on this quality assessment, five measurements were excluded from analysis (for details see Supplementary Information). In the resulting data set, Cramér-Rao Lower Bounds (CRLB) for Glu were $\leq$ $11 \%$ for all measurements. CRLB serve as quality criterion to determine the reliability of the fit for each metabolite and are considered reliable when $<20 \% 30$.

For exemplary spectrum and overall MRS data quality see Fig. 1 and Supplementary Table S3. All parameters for spectral quality are further presented per condition in the Supplementary Tables S4 - S7. Spectral quality between groups in the four different conditions did not differ $(P>0.2)$, thus enabled valid group comparisons between conditions (for statistics see Supplementary Tables S4 - S7).

\section{Glutamate concentration in the Nacc}

We found strongly decreased basal Glu concentrations in CAI $(P=0.0047, d=-0.89,95 \% \mathrm{CI}$ $[-1.47,-0.32]$ ) compared to HCI during the neutral film (Table 1 and Fig. 3). In accordance with the described raise of craving level in CAI, we also observed elevated Glu concentrations in the Nacc of CAI during the cocaine film. An interaction effect showed an increase in Glu levels from the neutral to the cocaine film in CAI, while in $\mathrm{HCI}$ a reduction was observed $(P=0.013 d \mathrm{CAI}=0.44,95 \%$ CI $[-0.12,1.00], d \mathrm{HCI}=-0.50,95 \%$ CI $[-1.03$, 0.02], Table 1 and Fig. 3). Also for Glx, a significant main effect of film was found, while trends were observed for the group and the interaction effect (for details see Supplementary Table S8). There was no significant correlation between Glu and craving levels in the cocaine film condition $(P>0.35)$.

\section{Pharmacological modulation}


Impaired glutamate homeostasis...

Contrary to expectations, the pharmacological challenge showed neither an effect on craving nor on Glu levels in CAI. However, the cue-induced Glu increase in CAI while taking placebo (see Table 1 and Fig. 3) was no longer observed in the N-AC condition. Unexpectedly, the N-AC challenge showed a strong effect on Glu in HCI $(P=0.024, d=$ $0.82,95 \%$ CI $[-1.36,-0.28], d$ calculated for neutral film placebo vs. N-AC), contrary to the expected effect on CAI, leading to an overall reduction in Glu across both conditions (Supplementary Table S9). For an overview of adverse events during study duration, see Supplementary Table S10.

\section{Discussion}

Research focussing on the role of the dopamine system in addiction has significantly advanced our understanding of the disease, whereas it has not resulted in effective treatments yet (26). Here, we evaluated alterations in the Glu-ergic homeostasis in the Nacc in CAI, since animal models suggested Glu imbalance in the Nacc to underlie addiction-like behaviour6. Therefore, we examined Glu concentrations in the Nacc of CAI by means of a dedicated ${ }_{1} \mathrm{H}-\mathrm{MRS}$ protocol. In accordance with animal models of addiction6, our data provide the first evidence in humans that $\mathrm{CA}$ is characterized by profound Glu-ergic shifts within the Nacc. We show that Glu concentrations in the Nacc were reduced in CAI in the withdrawal state relative to HCI, whereas Glu levels in CAI were amplified by visual and auditory cocaine-related cues reliably inducing craving symptoms. These results are consistent with alterations in Glu transmission described in substance-seeking reinstatement models in rodents, and indicate that accumbal Glu signalling is generally inhibited after chronic cocaine use and is accelerated during cue-exposure. This Glu imbalance is in line with, and might be explained by, molecular mechanisms that have been observed in animals exposed to cocaine. After withdrawal from cocaine, the expression of Glu transporters 1 and regulation via glial glutamate-cystine transporters are consistently down-regulated $9,31-33$. As extracellular Glu normally provides release-regulating tone on presynaptic metabotropic Glu receptors 2 and 3 (mGluR2/3)34, the signalling of mGluR2/3 is diminished following chronic cocaine administration9,35. Together, these adaptations in the Glu system lead to decreased basal Glu concentration in the extrasynaptic space of the Nacc core after chronic cocaine administration. In turn, during substance-seeking reinstatement, dysregulated Glu release combined with impaired Glu removal promotes an overflow of synaptically released Glu in the Nacc core9. Thus, our findings might reflect a common Glu-ergic dysfunction in the Nacc underlying the mammalian craving brain. 
However, $1 \mathrm{H}-\mathrm{MRS}$ does not allow differentiating intra- and extrasynaptic Glu, and therefore final inferences from Glu concentrations regarding directional Glu distribution within the different cellular compartments in humans are not possible yet. Spatial inferences are further restricted since voxel size and shape, which define the region wherein Glu is measured by $1 \mathrm{H}-$ MRS, cover the Nacc including both core and shell regions, whereas preclinical findings described Glu changes within the core only. On the contrary, this limited spatial specificity rather amplifies the probability for false negative results, not for false positives.

Given that the Nacc has been suggested to be also involved in salience detection 36 as well as in sleep-wake homeostasis37, the present Glu decrease observed in HCI while watching the cocaine film might reflect a decrease of salience of the audio-visual inputs over time together with a fatigue-driven Glu reduction, since the cocaine film was always shown after the neutral film and might constitute a stimulus with low salience for HCI. In CAI, on the other hand, the expected higher salience of cocaine-related cues38 together with heightened arousal might contribute to increased Glu levels during the cocaine film, analogue to animals showing accelerated Glu release in the Nacc in response to cues linked with incentive salience39.

Glu imbalance in CAI was not significantly modulated by the short-term intake of N-AC (Table 1). However, in the N-AC condition, the cue-induced Glu increase in CAI while taking placebo was no longer observed, whereas N-AC had no impact on craving ratings. Given that N-AC caused a significant reduction of Glu in the control group, which is in line with recent ${ }_{1} \mathrm{H}-\mathrm{MRS}$ Glu measures of the basal ganglia in a healthy population $40, \mathrm{~N}$-AC seems, in principle, capable of affecting the Glu system of the human brain. Further, recent findings have shown beneficial effects of long-term N-AC interventions of 25 days (as compared to our two-day administration) on problems related to CA41. Thus, an adjustment of the duration and potentially of the dose of N-AC intervention might be needed for reducing craving in CAI. Lastly, since the mechanisms of N-AC are far from being fully understood 42 , it remains challenging to interpret our unanticipated findings regarding the N$\mathrm{AC}$ administration. Thus, the impact of numerous other pharmacological compounds targeting the Glu system 43 should be further studied by means of a small-voxel $1 \mathrm{H}-\mathrm{MRS}$ approach as proposed here. We hypothesise that Glu levels within the human Nacc could serve as a non-invasive biomarker to predict individual treatment responses in future clinical trials. Namely, such a biomarker would allow identifying individuals who benefit the most from pharmacological interventions aiming at restoring disturbances within the Glu homeostasis in CAI, and thus would enable a personalized pharmacological treatment. 
Impaired glutamate homeostasis...

In sum, our findings contribute to the establishment of a translational framework of neurometabolic changes within the Glu system underlying CA across species that will enhance our understanding of addictive disorders, and, most importantly can thereby foster the development of urgently needed novel pharmacotherapies that target the Glu system.

Supplementary Information accompanies the paper on the Molecular Psychiatry's website (http://www.nature.com/mp). 


\section{References}

1 Asensio S, Romero MJ, Palau C, Sanchez A, Senabre I, Morales JL et al. Altered neural response of the appetitive emotional system in cocaine addiction: an fMRI Study. Addict Biol 2010; 15: 504-516.

2 Goldstein RZ, Alia-Klein N, Tomasi D, Zhang L, Cottone LA, Maloney T et al. Is decreased prefrontal cortical sensitivity to monetary reward associated with impaired motivation and self-control in cocaine addiction? Am J Psychiatry 2007; 164: 43-51.

3 Preller KH, Herdener M, Schilbach L, Stämpfli P, Vonmoos M, Ingold N et al. Functional changes of the reward system underlie blunted response to social gaze in cocaine users. Proc Natl Ac Sci USA 2014; 111: 2842-2847.

4 Kirschner M, Sladky R, Haugg A, Stämpfli P, Jehli E, Hodel M et al. Self-regulation of the dopaminergic reward circuit in cocaine users with mental imagery and neurofeedback. EBioMedicine 2018; 37: 489-498.

5 Haber SN, Knutson B. The Reward Circuit: Linking Primate Anatomy and Human Imaging. Neuropsychopharmacology 2009; 35: 4-26.

6 Kalivas PW. The glutamate homeostasis hypothesis of addiction. Nat rev Neurosci 2009; 10: 561-572.

7 Lüscher C, Malenka RC. Drug-evoked synaptic plasticity in addiction: from molecular changes to circuit remodeling. Neuron 2011; 69: 650-663.

8 Scofield MD, Kalivas PW. Astrocytic dysfunction and addiction: consequences of impaired glutamate homeostasis. Neuroscientist 2014; 20: 610-622.

9 Mulholland PJ, Chandler LJ, Kalivas PW. Signals from the fourth dimension regulate drug relapse. Trends Neurosci 2016; 39: 472-485.

10 McFarland K, Lapish CC, Kalivas PW. Prefrontal glutamate release into the core of the nucleus accumbens mediates cocaine-induced reinstatement of drug-seeking behavior. $J$ Neurosci 2003; 23: 3531-3537.

11 Quednow BB, Herdener M. Human pharmacology for addiction medicine: From evidence to clinical recommendations. Prog Brain Res 2016; 224: 227-250. 
12 Hulka LM, Scheidegger M, Vonmoos M, Preller KH, Baumgartner MR, Herdener M et al. Glutamatergic and neurometabolic alterations in chronic cocaine users measured with (1) H-magnetic resonance spectroscopy. Addict Biol 2014; 21: 205-217.

13 Schmaal L, Veltman DJ, Nederveen A, van den Brink W, Goudriaan AE. NAcetylcysteine Normalizes Glutamate Levels in Cocaine-Dependent Patients: A Randomized Crossover Magnetic Resonance Spectroscopy Study. Neuropsychopharmacology 2012; 37: 2143-2152.

14 Yang S, Salmeron BJ, Ross TJ, Xi Z-X, Stein EA, Yang Y. Lower glutamate levels in rostral anterior cingulate of chronic cocaine users - A (1)H-MRS study using TEaveraged PRESS at 3 T with an optimized quantification strategy. Psychiatry Res 2009; 174: $171-176$.

15 Martinez D, Slifstein M, Nabulsi N, Grassetti A, Urban NBL, Perez A et al. Imaging glutamate homeostasis in cocaine addiction with the metabotropic glutamate receptor 5 positron emission tomography radiotracer $[(11) \mathrm{C}] \mathrm{ABP} 688$ and magnetic resonance spectroscopy. Biol Psychiatry 2014; 75: 165-171.

16 de Graaf RA. In Vivo NMR Spectroscopy. 2nd ed. John Wiley \& Sons Ltd., Chichester: Chichester, UK, 2007.

17 Neto LL, Oliveira E, Correia F, Ferreira AG. The human nucleus accumbens: where is it? A stereotactic, anatomical and magnetic resonance imaging study. Neuromodulation 2008; 11: 13-22.

18 Dreher W, Leibfritz D. New method for the simultaneous detection of metabolites and water in localized in vivo $1 \mathrm{H}$ nuclear magnetic resonance spectroscopy. Magn Reson Med 2005; 54: 190-195.

19 Hock A, MacMillan EL, Fuchs A, Kreis R, Boesiger P, Kollias SS et al. Non-watersuppressed proton MR spectroscopy improves spectral quality in the human spinal cord. Magn Reson Med 2012; 69: 1253-1260.

20 MacMillan EL, Chong DGQ, Dreher W, Henning A, Boesch C, Kreis R. Magnetization exchange with water and $\mathrm{T} 1$ relaxation of the downfield resonances in human brain spectra at 3.0 T. Magn Reson Med 2011; 65: 1239-1246. 
21 Zoelch N, Hock A, Henning A. Quantitative magnetic resonance spectroscopy at 3T based on the principle of reciprocity. NMR Biomed 2018; 55: e3875.

22 Yagen B, Hernandez O, Bend JR, Cox RH. Synthesis and relative stereochemistry of the four mercapturic acids derived from styrene oxide and $\mathrm{N}$-acetylcysteine. Chem Biol Interact 1981; 34: 57-67.

23 McClure EA, Gipson CD, Malcolm RJ, Kalivas PW, Gray KM. Potential role of Nacetylcysteine in the management of substance use disorders. CNS Drugs 2014; 28: 95106.

24 American Psychiatric Association. Diagnostic and Statistical Manual of Mental Disorders, Fourth Edition, Text Revision. American Psychiatric Association Press: Washington, DC, 2000.

25 Provencher SW. Estimation of metabolite concentrations from localized in vivo proton NMR spectra. Magn Reson Med 1993; 30: 672-679.

26 Friston KJ, Ashburner JT, Kiebel SJ, Nichols TE, Penny WD. Statistical Parametric Mapping. 1st ed. Academic Press, London: London, 2006.

27 Mardikian PN, LaRowe SD, Hedden S, Kalivas PW, Malcolm RJ. An open-label trial of $\mathrm{N}$-acetylcysteine for the treatment of cocaine dependence: A pilot study. Prog NeuroPsychopharmacol Biol Psychiatry 2007; 31: 389-394.

28 Holdiness MR. Clinical pharmacokinetics of N-acetylcysteine. Clin Pharmacokinet 1991; 20: 123-134.

29 Brown H, Prescott R. Applied Mixed Models in Medicine. John Wiley \& Sons, Chichester: Chichester, UK, 2006.

30 Oz G, Alger JR, Barker PB, Bartha R, Bizzi A, Boesch C et al. Clinical proton MR spectroscopy in central nervous system disorders. Radiology 2014; 270: 658-679.

31 Knackstedt LA, Melendez RI, Kalivas PW. Ceftriaxone restores glutamate homeostasis and prevents relapse to cocaine seeking. Biol Psychiatry 2010; 67: 81-84.

32 Trantham-Davidson H, LaLumiere RT, Reissner KJ, Kalivas PW, Knackstedt LA. 
Ceftriaxone normalizes nucleus accumbens synaptic transmission, glutamate transport, and export following cocaine self-administration and extinction training. J Neurosci 2012; 32: 12406-12410.

33 Reissner KJ, Gipson CD, Tran PK, Knackstedt LA, Scofield MD, Kalivas PW. Glutamate transporter GLT-1 mediates N-acetylcysteine inhibition of cocaine reinstatement. Addict Biol 2015; 20: 316-323.

34 Moran MM, McFarland K, Melendez RI, Kalivas PW, Seamans JK. Cystine/glutamate exchange regulates metabotropic glutamate receptor presynaptic inhibition of excitatory transmission and vulnerability to cocaine seeking. J Neurosci 2005; 25: 6389-6393.

35 Bowers MS, McFarland K, Lake RW, Peterson YK, Lapish CC, Gregory ML et al. Activator of $\mathrm{G}$ protein signaling 3: a gatekeeper of cocaine sensitization and drug seeking. Neuron 2004; 42: 269-281.

36 Zaehle T, Bauch EM, Hinrichs H, Schmitt FC, Voges J, Heinze H-J et al. Nucleus accumbens activity dissociates different forms of salience: evidence from human intracranial recordings. J Neurosci 2013; 33: 8764-8771.

37 Léna I, Parrot S, Deschaux O, Muffat-Joly S, Sauvinet V, Renaud B et al. Variations in extracellular levels of dopamine, noradrenaline, glutamate, and aspartate across the sleep--wake cycle in the medial prefrontal cortex and nucleus accumbens of freely moving rats. J Neurosci Res 2005; 81: 891-899.

38 Robinson TE, Berridge KC. The neural basis of drug craving: an incentive-sensitization theory of addiction. Brain Res Rev 1993; 18: 247-291.

39 Batten SR, Pomerleau F, Quintero J, Gerhardt GA, Beckmann JS. The role of glutamate signaling in incentive salience: second-by-second glutamate recordings in awake Sprague-Dawley rats. J Neurochem 2018; 145: 276-286.

40 O'Gorman Tuura R, Warnock G, Ametamey S, Treyer V, Noeske R, Buck A et al. Imaging glutamate redistribution after acute $\mathrm{N}$-acetylcysteine administration: A simultaneous PET/MR study. NeuroImage 2019; 184: 826-833.

41 Schulte MHJ, Wiers RW, Boendermaker WJ, Goudriaan AE, van den Brink W, van 
Impaired glutamate homeostasis...

Deursen DS et al. The effect of $\mathrm{N}$-acetylcysteine and working memory training on cocaine use, craving and inhibition in regular cocaine users: correspondence of lab assessments and Ecological Momentary Assessment. Addict Behav 2017; 79: 24-31.

42 Womersley JS, Townsend DM, Kalivas PW, Uys JD. Targeting redox regulation to treat substance use disorder using N-acetylcysteine. Eur J Neurosci 2019; 50: 2538-2551.

43 Olive MF, Cleva RM, Kalivas PW, Malcolm RJ. Glutamatergic medications for the treatment of drug and behavioral addictions. Pharmacol Biochem Behav 2012; 100: 801-810. 
Impaired glutamate homeostasis...

\section{Acknowledgments}

We thank Johanna Klar, Nathalie Rieser, Martina Riva, and Colette

Steinegger for assistance in data collection and Katrin Preller for support with questionnaire programming. This project was supported by a grant from the Zurich Center for Integrative Human Physiology, University of Zurich.

\section{Conflict of interests}

Unrelated to this study, M.H. has received speaker fees from Lundbeck, and has served as a consultant for and received research support from Novartis. The other authors declare that they have no conflict of interest. 


\section{A}
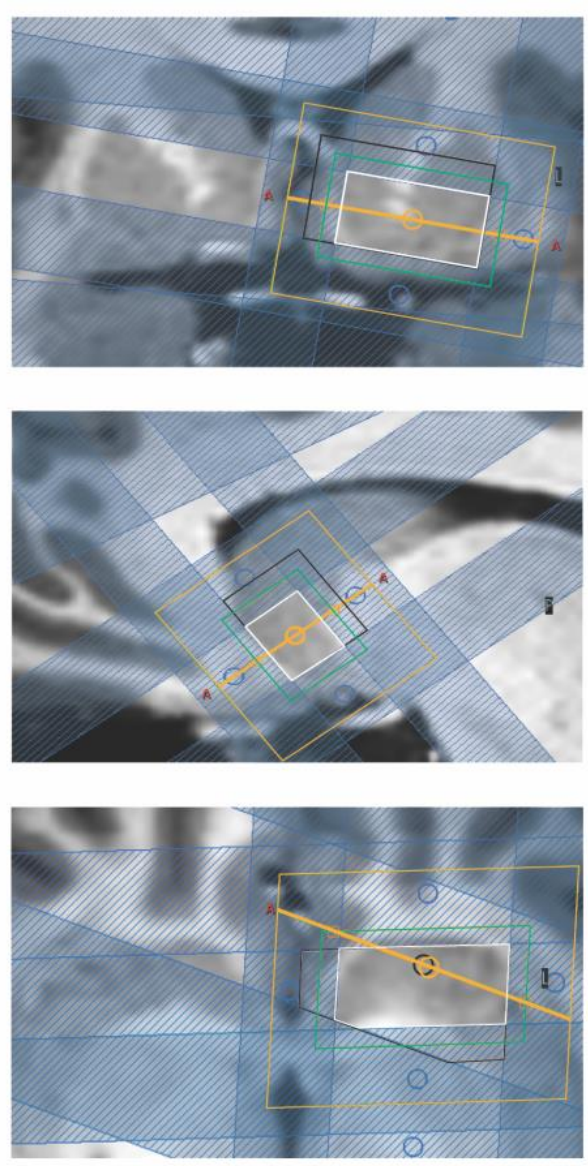

B

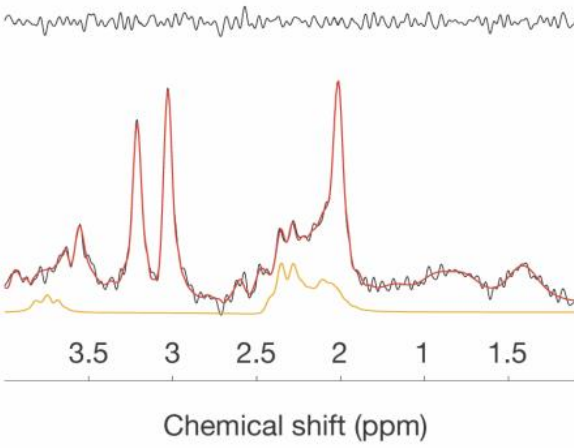

Figure 1. Proton magnetic resonance spectroscopy (1H-MRS) assessment of glutamate concentration in the nucleus accumbens. (A) Coronal, sagittal and axial slice of T1-weighted images of the brain illustrating the tilted position of the $1 \mathrm{H}-\mathrm{MRS}$ acquisition voxel centred on the left nucleus accumbens. The effective voxel size (white; $9.4 \times 18.8 \times 8.4 \mathrm{~mm} 3$ ) results from the overlap localized volumes of lactate/lipid (black) and water (green) defined by saturation bands (blue; for details see Supplementary Information). The shim box is indicated in orange. (B) Exemplary single spectrum measured therein (black), LCModel fit (red), fitresiduals (above; black), and glutamate and glutamine extracted (yellow). 

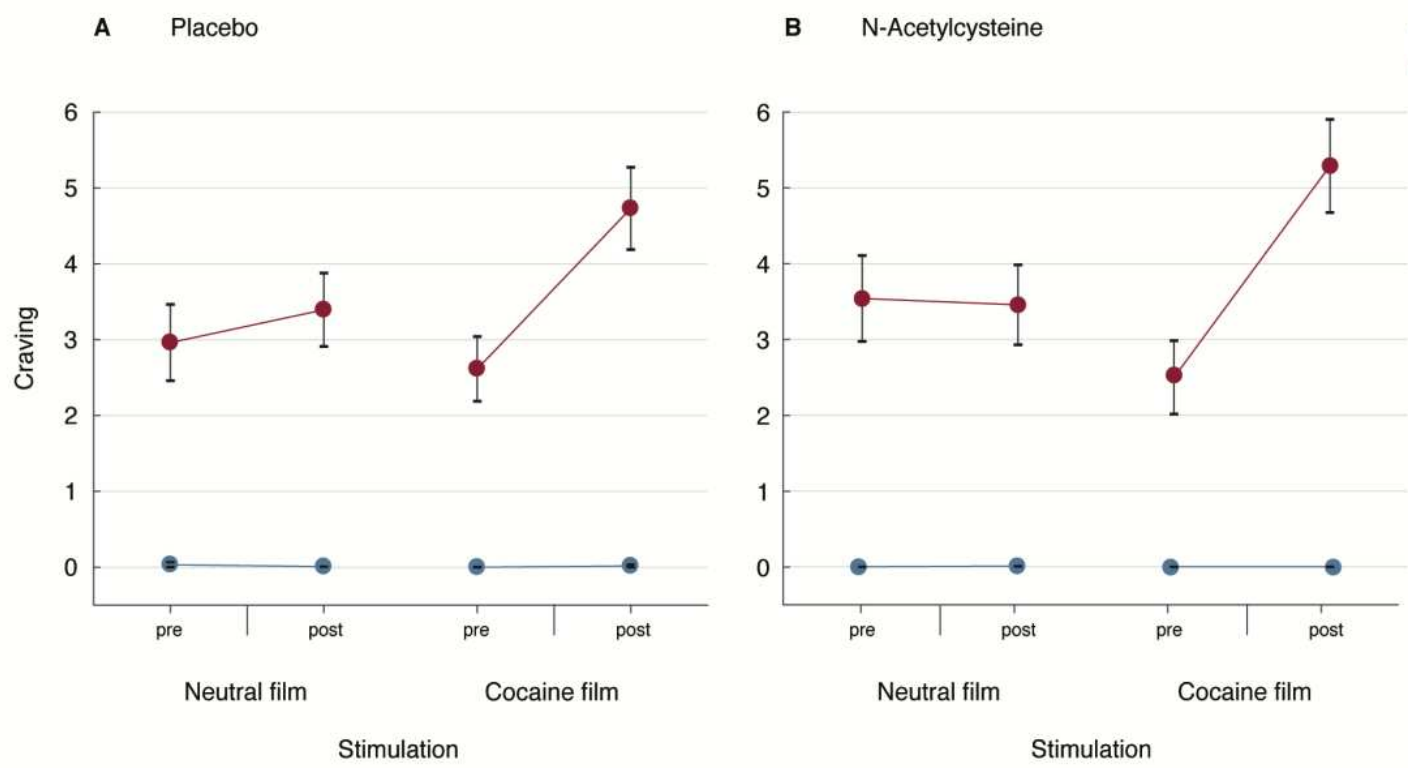

Figure 2. Craving as a function of cocaine-cue stimulation. Plot of mean craving ratings per group before and after the presentation of a neutral and a cocaine film represented as dots with standard error bars; blue $=$ healthy control individuals, red $=$ cocaine-addicted individuals. A: Placebo condition: A random coefficient model showed significantly higher craving ratings in cocaine-addicted individuals $(P<0.0001, \mathrm{~N}=26)$ at all measurement points compared to healthy control individuals $(\mathrm{N}=30)$, and significantly increased craving ratings after the cocaine film in cocaine-addicted individuals compared to their ratings before the cocaine film $(P=0.0002)$. B: N-acetylcysteine $(\mathrm{N}-\mathrm{AC})$ condition: No significant effects were observed for either group. For statistics, see Table 1. 

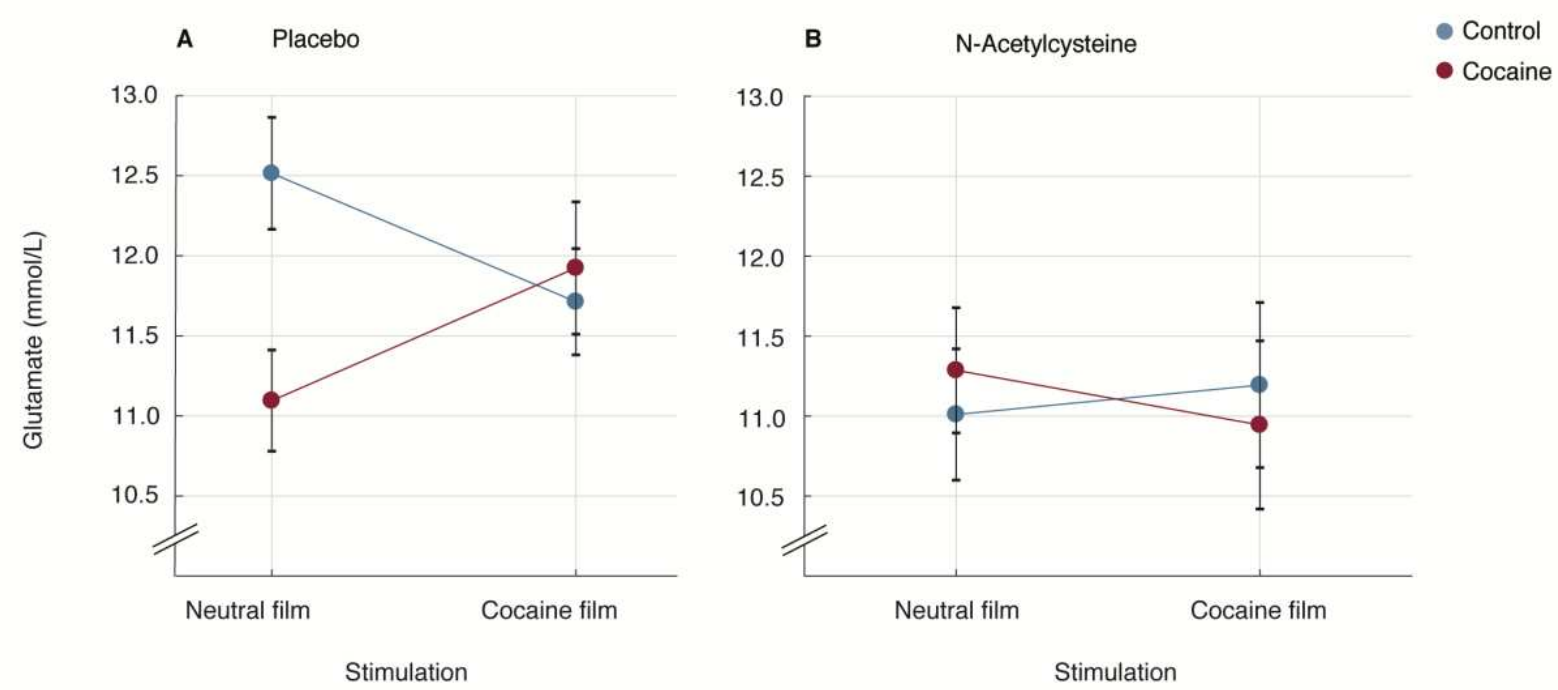

Figure 3. Impact of cocaine-cue stimulation on glutamate levels within the nucleus accumbens in cocaine addiction. Plot of mean glutamate concentrations in millimoles per litre $(\mathrm{mmol} / \mathrm{L})$ per group during presentation of a neutral and a cocaine film represented as dots with standard error bars; blue = healthy control individuals, red = cocaine-addicted individuals. A: Placebo condition: A random coefficient model showed significantly lower basal glutamate levels (neutral film, $P=0.0047)$ in cocaine-addicted individuals $(\mathrm{N}=26)$ compared to healthy control individuals $(\mathrm{N}=30)$, and significantly increased cue-induced glutamate levels (cocaine film) compared to baseline levels (neutral film, $P=0.013$ ). B: Nacetylcysteine (N-AC) condition: No significant effect was observed in cocaine-addicted individuals, while a significant main effect for N-AC was found for healthy control individuals. For statistics, see Table 1. 
Impaired glutamate homeostasis...

\begin{tabular}{|c|c|c|c|c|}
\hline \multirow[b]{3}{*}{ Fixed effects } & \multicolumn{4}{|l|}{ Dependent variables } \\
\hline & \multicolumn{2}{|l|}{ Craving levels } & \multicolumn{2}{|l|}{ Glutamate ratings } \\
\hline & estimate \pm s.e.m. & $P$ Value & estimate \pm s.e.m. & $P$ Value \\
\hline Intercept $(\mathrm{HCl})$ & $0.03 \pm 0.34$ & 0.92 & $12.62 \pm 0.35$ & $<0.0001^{* * * *}$ \\
\hline CAI (vs. $\mathrm{HCl}$ ) & $3.02 \pm 0.50$ & $<0.0001^{* \star * *}$ & $-1.51 \pm 0.51$ & $0.0047^{* *}$ \\
\hline Cocaine film & $-0.02 \pm 0.28$ & 0.95 & $-0.91 \pm 0.46$ & 0.053 \\
\hline CAI $\times$ cocaine & $1.69 \pm 0.41$ & $0.0002^{\star \star \star}$ & $1.72 \pm 0.67$ & $0.013^{*}$ \\
\hline Residual & 1.12 & & 2.95 & \\
\hline Number of parameters & 5 & & 5 & \\
\hline Number of participants & $56(26 \mathrm{CAl} / 30 \mathrm{HCl})$ & & $56(26 \mathrm{CAl} / 30 \mathrm{HCl})$ & \\
\hline Number of data points & 108 & & 107 & \\
\hline
\end{tabular}

Table 1. Group differences and influence of cocaine film displayed by estimates and standard errors (s.e.m.) for main effects (CAI, cocaine film) in reference to HCI (intercept), and by the interaction term (CAI x cocaine film).

$\mathrm{CAI}=$ cocaine-addicted individuals

$\mathrm{HCI}=$ healthy control individuals

$* P<.05 ; * * P<.01 ; * * * P<.001 * * * * P<0.0001$ 


\section{Supplementary Information}

\section{Impaired glutamate homeostasis in the nucleus accumbens in human cocaine addiction}

Etna J. E. Engeli, MSc1*, Niklaus Zoelch, $\mathrm{PhD}_{2,3,4}$, Andreas Hock, PhD 2,4, Carlos Nordt, PhD 1, Lea M. Hulka, PhD 1, Matthias Kirschner MD1,5, Milan Scheidegger, MD, PhD 4, Fabrizio Esposito, Prof.6, Markus R. Baumgartner, PhD7, Anke Henning, Prof.2,8,9,10, Erich Seifritz Prof.4,8,11, Boris B. Quednow Prof.8,11,12, Marcus Herdener, MD1

${ }_{1}$ Centre for Addictive Disorders, Department of Psychiatry, Psychotherapy and Psychosomatics, Psychiatric Hospital, University of Zurich, Switzerland.

2 Institute for Biomedical Engineering, University of Zurich and Swiss Federal Institute of Technology Zurich, Switzerland.

3 Institute of Forensic Medicine, University of Zurich, Switzerland.

4 Department of Psychiatry, Psychotherapy and Psychosomatics, Psychiatric Hospital, University of Zurich, Switzerland.

5 Montreal Neurological Institute, McGill University, Montreal, Quebec, Canada.

6 Department of Medicine, Surgery and Dentistry, Scuola Medica Salernitana, University of Salerno, Baronissi, Salerno, Italy.

${ }_{7}$ Centre for Forensic Hair Analytics, Zurich Institute of Forensic Medicine, University of Zurich, Switzerland.

8 Zurich Centre for Integrative Human Physiology, University of Zurich, Switzerland.

9 Max-Planck-Institute for Biological Cybernetics, Tuebingen, Germany.

10 Institute of Physics, Ernst-Moritz-Arndt University Greifswald, Germany.

11 Neuroscience Centre Zurich, University of Zurich and Swiss Federal Institute of Technology Zurich, Switzerland.

12 Experimental and Clinical Pharmacopsychology, Department of Psychiatry, Psychotherapy and Psychosomatics, Psychiatric Hospital, University of Zurich, Switzerland.

* Corresponding author:

Etna J. E. Engeli, MSc

Centre for Addictive Disorders

Department of Psychiatry, Psychotherapy and Psychosomatics,

Psychiatric Hospital, University of Zurich

Selnaustrasse 9, CH-8001 Zurich, Switzerland

Tel.: +41 (0)44 2055800

E-Mail: etna.engeli@bli.uzh.ch 


\section{Participants}

\section{Sample size}

The initial definition of sample size $\mathrm{N}=30 / 30$ resulted from a power analysis ( $80 \%$ power, $d$ $=0.8, P<0.5)$ based on previous $1 \mathrm{H}-\mathrm{MRS}$ measures of glutamate $(\mathrm{Glu})$ in prefrontal areas of cocaine users (Hulka, 2014), and an anticipated dropout rate of $30 \%$. Since data loss was higher than anticipated, 36 cocaine-addicted individuals (CAI) and 33 healthy control individuals (HCI) were randomised into the study (03.2015 - 04.2016). In 40.6\% (CAI: 17; HCI: 10) of all participants at least one of four data sets were missing, and of 23.2\% (CAI: 12; HCI: 4) of all participants the complete data set was missing or could not be used.

Two CAI aborted the study participation due to unexpected sensation of claustrophobia when entering the magnetic resonance scanner. Four participants (three HCI; one CAI) aborted the study participation during or after the first proton magnetic resonance spectroscopy ( $1 \mathrm{H}-\mathrm{MRS})$ session because of demotivation, two participants (one HCI; one CAI) citing scheduling difficulties, two participants (one HCI; one CAI) because of acute illness and one HCI due to discomfort in the magnetic resonance scanner (neck pain). Two CAI did not attend their study sessions for unknown reasons. Further, two CAI began novel pharmacological treatments (methylphenidate) during the study participation, either prior to the first or to the second $1 \mathrm{H}$ MRS session. The hair analysis of one CAI revealed a mean cocaine concentration during the last six months slightly below the threshold of reliable detection $(<500 \mathrm{mg} / \mathrm{mg})$. This clearly indicates a merely occasional cocaine use at most, thus, likely below the predefined minimum of one gram per week. Further, the hair analysis of one HCI unveiled concealed pharmacological treatment for epilepsy targeting the GABA and Glu system and was therefore excluded. Two CAI and one HCI were sleeping during the $1 \mathrm{H}-\mathrm{MRS}$ measurement and were therefore excluded. Two ${ }_{1} \mathrm{H}-\mathrm{MRS}$ measurements were excluded owing to subject motion of > $5 \mathrm{~mm}$, two measurements due to $\mathrm{SNR} \leq 10$ and one measurement due to linewidth $>12.7 \mathrm{~Hz}$. In total, five $1 \mathrm{H}-\mathrm{MRS}$ measurements were excluded based on poor data quality (two HCI; three CAI). Finally, two CAI and one HCI could not be measured at the second session as of technical issues with the magnetic resonance scanner.

After all affected measurements were excluded, data from $26 \mathrm{CAI}$ and $30 \mathrm{HCI}$ were included in the analysis, unless stated otherwise. The participants with adequate $1 \mathrm{H}-\mathrm{MRS}$ data acquired in the placebo condition served as a reference for the sample selection for all other analysis. 
Impaired glutamate homeostasis...

\section{Sample characteristics}

As expected and in line with previous studies1,2, CAI reported significantly more symptoms in the Beck Depression Inventory (BDI) 3 and the attention-deficit hyperactivity disorder (ADHD) self-rating scale (ADHD-SR)4, and scored higher on the Fagerström Test for Nicotine Dependence (FTND) 5 than healthy control individuals. For detailed characterisation, see Table S1.

\section{Methods}

\section{Study design}

This study was designed as a randomized, double-blind, placebo-controlled, crossover trial (clinicalTrial.gov identifier: NCT02626494). The complete investigation consisted of three measurement time points and was conducted at the Psychiatric Hospital of the University of Zurich (for illustration of study design and participant allocation see Figure S1). The first measurement session was conducted to examine demographic data, psychiatric disorders, substance use, and personality traits. Psychologists screened all participants for the presence of DSM-IV axis I psychiatric disorders using the Mini-International Neuropsychiatric Interview6 as a structured diagnostic psychiatric interview. Substance use was assessed by means of a structured and standardized Interview for Psychotropic Drug Consumption7, and hair samples were collected to objectively characterize substance use over the past three to six months.

To match the two groups, demographic information and smoking habits were collected with a computer-based questionnaire. Additionally, the Mehrfachwahl Wortschatz Intelligenztest (MWT-B) was applied to capture premorbid verbal intelligences. For further characterisation of the sample, all participants completed standardized questionnaires concerning individual traits. The $\mathrm{BDI}_{3}$ and the ADHD-SR4 measured current symptoms of depression and ADHD, respectively. The Barratt Impulsiveness Scale (BIS-11)9 was applied to estimate trait impulsivity. The Fagerström Test for Nicotine Dependence (FST) 5 was used to determine severity of nicotine dependence, and the Alcohol Use Disorder Identification Test (AUDIT)10 to assess the risk for alcohol abuse.

After the assessment session, two identical ${ }_{1} \mathrm{H}-\mathrm{MRS}$ measurement sessions on two separate days followed to allow placebo-controlled investigation of a short-term $\mathrm{N}$-acetylcysteine $(\mathrm{N}$ AC) challenge. Both $1 \mathrm{H}-\mathrm{MRS}$ sessions started in the morning. According to the procedures in preclinical studies, all participants were asked to refrain from illicit substances for three days, 
Impaired glutamate homeostasis...

from alcohol for two days, and usual tobacco consumption was allowed until one hour prior to the $1 \mathrm{H}-\mathrm{MRS}$ scans.

\section{H-MRS data acquisition}

Prior to each of four blocks of a scan, all preparation steps, including power optimization, centre frequency, shimming, receiver gain optimization, were repeatedly carried out to take potential scanner drifts into account. After each block, voxel position was revised by acquiring an axial T2-weighted image, since even small participant movements lead to incorrect positions for small voxels. If a change of voxel position (> $5 \mathrm{~mm}$ ) was detected and if the experimental time schedule allowed it, the voxel placement was repositioned for the next block. This block replaced the previous block affected by participant movement. If repetition of the block was not possible, spectra with subject motion $>5 \mathrm{~mm}$ were excluded from analysis.

Further, to avoid estimations of the water concentration and relaxation properties in the investigated tissue, a calibration strategy based on a reference signal acquired in a phantom with known concentrations was applied. Necessary corrections for sensitivity and coil loading changes between in-vivo and phantom measurement were performed11. In this approach, the optimal radiofrequency pulse voltage, as a measure of coil sensitivity and coil loading, is automatically determined by using the selective power optimization 12 prior to each measurement. After correction, external referencing allows estimation of absolute concentrations of metabolites. Additional corrections for potential deviations of the transmit sensitivity from the receptions sensitivity 11 were omitted due to the small distance between voxel position and the central axis of the coil. Segmentation of T1-weighted images within acquisition voxel using SPM813 revealed mean tissue composition of grey matter ( 77\%), white matter $(\sim 23 \%)$ and cerebrospinal fluid $(0 \%)$ did not differ between groups (NCAI $=95$, NHCI $=110$, independent t-test, 2-tailed, $P>0.6$,). Given that there was no cerebrospinal fluid fraction within the voxel, it appears that the applied small voxel allowed an unprecedented precise assessment of the Nacc.

\section{H-MRS quality}

Table S3 presents the achieved $1 \mathrm{H}-\mathrm{MRS}$ data quality across groups and over all conditions after exclusion of six measurements due to poor quality as described in detail in Sample size. To test whether there are differences in spectral quality between groups, we compared the ${ }_{1} \mathrm{H}-\mathrm{MRS}$ quality parameters of each condition between the groups. All parameters were comparable as 
shown in Table S4 - S7. Further to ensure, that the within-group Glu difference in CAI between neutral and cocaine film did not result from quality inequalities, a post-hock ANOVA was performed. The results show that $1 \mathrm{H}-\mathrm{MRS}$ quality parameters of CAI did not differ between the films in the placebo condition ( $\mathrm{N}=24 / 26$, one-way ANOVA, $P>0.2)$.

Glu is synthesised by glutamine and their chemical similarity leads to overlapping magnetic resonance spectra14, which impedes unambiguous independent quantification of Glu and glutamine at field strength $<7$ Tesla. To date, this impediment has not been fully overcome, and thus remains a challenge of in vivo Glu quantification. Nonetheless, we strived to address this issue by tailoring a dedicated $1 \mathrm{H}-\mathrm{MRS}$ protocol for low concentrated metabolites and small brain regions. We performed further a diligent quality assessment of the achieved data following consensus guidelines15, which were clearly fulfilled despite size and localisation of the applied voxel. Moreover, these considerations apply to both CAI and HCI to the same extent, therefore enabling a valid comparison between groups. Finally, the achieved results coincide with our apriori hypotheses, and thus with the well-studied animal model.

\section{Pharmacological challenge}

The use of N-AC was authorized by Swissmedic, the Swiss agency for therapeutic products. The study compounds were manufactured, randomised and blinded by the cantonal pharmacy of Zurich following Good Clinical Practice guidelines. Block randomisation of the study compound was implemented for the verum-placebo sequence to ensure a balance of the challenge order within groups. Forty study compounds for each group were prepared by the cantonal pharmacy of Zurich in sequentially numbered containers. During the study duration, both participants and investigators were blinded for the sequence allocation. It remained concealed until the last visit of the last patient was completed and was then revealed to the investigators by the cantonal pharmacy of Zurich for data analysis.

To increase the compliance of participants, they received a personalised study schedule, a medication study diary and a reminder by a study investigator. At the beginning of every visit, participants were casually asked about their well-being. During the entire study duration, no severe and no unexpected adverse events were reported. All adverse events were mild to moderate and contained previously described side effects, mainly on the gastrointestinal system. Overall, two participants (3.57\%) reported adverse events in the placebo condition, while five participants $(9.26 \%)$ reported adverse events in the N-AC condition. A detailed overview of adverse events is given in the Table S10. 
Impaired glutamate homeostasis...

\section{Urine screening and hair analysis}

Adherence with instructions regarding abstinence of psychoactive substances was monitored by qualitative urine analyses. Urine samples of 12 and $13 \mathrm{CAI}$, respectively, were positive for cocaine at the $1 \mathrm{H}-\mathrm{MRS}$ sessions in the placebo and the N-AC condition (for details, see Table S2). Since metabolites of psychoactive substances can be detectable in urine for more than three days in regular users, we included those participants with positive screening results into the analyses and controlled for its effect on Glu and craving. All urine samples of HCI were negative.

Quantification of the consumption of psychoactive substances over the past three and up to six months (depending on hair length) was performed by hair toxicology analyses. Proximal hair segments were analysed with liquid chromatography-tandem mass spectrometry based on a method by Rust and colleagues16. Overall, results confirmed regular use of cocaine and clear dominance of cocaine in CAI over other psychoactive substances (for details, see Table S2). Two hair analyses lead to data exclusion, for details see Sample size. 


\section{References}

1 Vonmoos M, Hulka LM, Preller KH, Jenni D, Schulz C, Baumgartner MR et al. Differences in self-reported and behavioral measures of impulsivity in recreational and dependent cocaine users. Drug Alcohol Depend 2013; 133: 61-70.

2 Ersche KD, Turton AJ, Chamberlain SR, Müller U, Bullmore ET, Robbins TW. Cognitive dysfunction and anxious-impulsive personality traits are endophenotypes for drug dependence. American J Psychiatry 2012; 169: 926-936.

3 Beck AT, Ward CH, Mendelson M, Mock J, Erbaugh J. An inventory for measuring depression. Arch Gen Psychiatry 1961; 4: 561-571.

4 Rösler M, Retz W, Retz-Junginger P, Thome J, Supprian T, Nissen T et al. Tools for the diagnosis of attention-deficit/hyperactivity disorder in adults. Self-rating behaviour questionnaire and diagnostic checklist. Nervenarzt 2004; 75: 888-895.

5 Heatherton TF, Kozlowski LT, Frecker RC, Fagerström KO. The Fagerström Test for Nicotine Dependence: a revision of the Fagerström Tolerance Questionnaire. Br J Addict 1991; 86: 1119-1127.

6 Sheehan DV, Lecrubier Y, Sheehan KH, Amorim P, Janavs J, Weiller E et al. The MiniInternational Neuropsychiatric Interview (M.I.N.I.): the development and validation of a structured diagnostic psychiatric interview for DSM-IV and ICD-10. J Clin Psychiatry 1998; 59 Suppl 20: 22-33.

7 Quednow BB, Kühn K-U, Hoenig K, Maier W, Wagner M. Prepulse Inhibition and Habituation of Acoustic Startle Response in Male MDMA ('Ecstasy') Users, Cannabis Users, and Healthy Controls. Neuropsychopharmacology 2004; 29: 982-990.

8 Lehrl S, Triebig G, Fischer B. Multiple choice vocabulary test MWT as a valid and short test to estimate premorbid intelligence. Acta Neurol Scand 1995; 91: 335-345.

9 Patton JH, Stanford MS, Barratt ES. Factor structure of the Barratt impulsiveness scale. J Clin Psychol 1995; 51: 768-774.

10 Saunders JB, Aasland OG, Babor TF, la Fuente de JR, Grant M. Development of the Alcohol Use Disorders Identification Test (AUDIT): WHO Collaborative Project on 
Early Detection of Persons with Harmful Alcohol Consumption--II. Addiction 1993; 88: 791-804.

11 Zoelch N, Hock A, Henning A. Quantitative magnetic resonance spectroscopy at 3T based on the principle of reciprocity. NMR Biomed 2018; 55: e3875.

12 Versluis MJ, Kan HE, van Buchem MA, Webb AG. Improved signal to noise in proton spectroscopy of the human calf muscle at $7 \mathrm{~T}$ using localized B1 calibration. Magn Reson Med 2010; 63: 207-211.

13 Friston KJ, Ashburner JT, Kiebel SJ, Nichols TE, Penny WD. Statistical Parametric Mapping. 1st ed. Academic Press, London: London, 2006.

14 Govindaraju V, Young K, Maudsley AA. Proton NMR chemical shifts and coupling constants for brain metabolites. NMR Biomed 2000; 13: 129-153.

15 Oz G, Alger JR, Barker PB, Bartha R, Bizzi A, Boesch C et al. Clinical proton MR spectroscopy in central nervous system disorders. Radiology 2014; 270: 658-679.

16 Rust KY, Baumgartner MR, Meggiolaro N, Kraemer T. Detection and validated quantification of 21 benzodiazepines and 3 'z-drugs' in human hair by LC-MS/MS. Forensic Sci Int 2012; 215: 64-72. 

a) Assessment
b) ${ }^{1} \mathrm{H}-\mathrm{MRS}$
c) ${ }^{1} \mathrm{H}-\mathrm{MRS}$

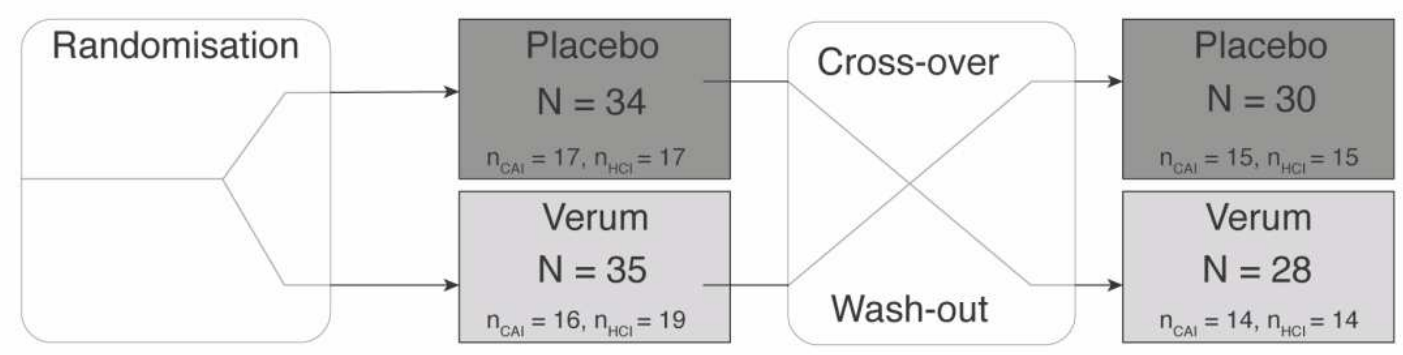

Figure S1. Illustration of study design and participant allocation. The study was performed in a randomized placebo-controlled double-blind cross-over design. a) After the assessment of eligibility criteria, participants were randomly assigned to an intervention order. b) The first proton magnetic resonance spectroscopy ( $1 \mathrm{H}-\mathrm{MRS})$ was performed under either placebo or verum ( $\mathrm{N}$-acetylcysteine). To rule out potential carry-over effects, a wash-out period of 14 days ( \pm 4 days) followed the first $1 \mathrm{H}-\mathrm{MRS}$ session/compound intake. c) The ${ }_{1} \mathrm{H}-\mathrm{MRS}$ session was repeated and the other compound was administered. Allocation ratios per conditions are indicated over all participants and separately for the groups.

$\mathrm{CAI}=$ Cocaine-addicted individuals

$\mathrm{HCI}=$ Healthy control individuals 
Impaired glutamate homeostasis...

Table S1. Description and comparison of demographic data within and between groups

\begin{tabular}{|c|c|c|c|c|c|}
\hline & Healthy control individuals & Cocaine-addicted individuals & & & \\
\hline & & & Value & df & $P$ Value \\
\hline Male/female & $19 / 11$ & $19 / 7$ & $\chi^{2}=0.61$ & 1 & 0.436 \\
\hline Age in years & $29.50 \pm 5.76$ & $29.94 \pm 5.99$ & $t=0.28$ & 54 & 0.785 \\
\hline Years of education & $10.02 \pm 1.42$ & $9.85 \pm 2.49$ & $t=-0.32$ & 54 & 0.750 \\
\hline Verbal IQ & $105.38 \pm 12.06$ & $101.15 \pm 9.91$ & $t=-1.29$ & 47 & 0.202 \\
\hline Smoker/non-smoker & $23 / 7$ & $24 / 2$ & FET & & 0.109 \\
\hline FTND sum score (0-10) & $1.30 \pm 1.97$ & $3.85 \pm 2.72$ & $t=4.05$ & 54 & $0.0002^{* * *}$ \\
\hline AUDIT sum score (0-32) & $4.80 \pm 2.80$ & $10.54 \pm 6.72$ & $t=4.28$ & 54 & $0.00008^{\star \star \star *}$ \\
\hline ADHD-SR sum score $(0-54)$ & $8.50 \pm 6.32$ & $13.54 \pm 8.85$ & $t=2.48$ & 54 & $0.017^{*}$ \\
\hline BDI sum score $(0-63)$ & $3.10 \pm 3.39$ & $7.62 \pm 7.82$ & $t=2.87$ & 54 & $0.006^{\star *}$ \\
\hline
\end{tabular}

Table S1. Means \pm standard deviations of demographic data per group. Groups were matched on age, sex, verbal intelligence quotient (IQ) and smoking status. Sample size N for cocaineaddicted individuals (CAI) and healthy control individuals (HCI), unless stated otherwise:

$\mathrm{NCAI}=26 \mathrm{NHCI}=30$.

${ }^{\circ}$ Verbal IQ was assessed with a test only applicable in German native speaking participants:

$\mathrm{NCAI}=20 \mathrm{NHCI}=29$

FTND: Fagerström Test of Nicotine Dependence

ADHD-SR: Attention-Deficit/Hyperactivity Disorder Self-Rating Scale

BDI: Beck Depression Inventory

$\chi 2: \chi^{2-t e s t}$ for frequency data

$\mathrm{t}$ : independent $\mathrm{t}$-test

FEZ: Fisher's exact test one-sided.

$* P<0.05, * * P<0.01, * * * P<0.001, * * * * P<0.0001$ 
Table S2. Description and comparison of psychoactive substance use within and between groups

\begin{tabular}{|c|c|c|c|c|c|}
\hline & $\begin{array}{c}\text { Cocaine-addicted } \\
\text { individuals }\end{array}$ & $\begin{array}{c}\text { Healthy control } \\
\text { individuals }\end{array}$ & & & \\
\hline & \multicolumn{2}{|c|}{ mean \pm s.d. } & Value & $\mathrm{df}$ & $P$ Value \\
\hline \multicolumn{6}{|l|}{ Cocaine } \\
\hline Grams/week & $5.52 \pm 10.03$ & 0 & $\mathrm{tw}=2.81$ & 25 & $0.010^{*}$ \\
\hline Frequency/week ${ }^{\circ}$ & $3.40 \pm 1.90$ & 0 & $\mathrm{tw}=9.00$ & 24 & $<0.0001^{* * * *}$ \\
\hline Cumulative dose (grams/lifetime) & $1782.56 \pm 2378.46$ & 0 & $\mathrm{tw}=3.82$ & 25 & $0.0008^{* * *}$ \\
\hline Years of use & $6.31 \pm 4.01$ & 0 & $\mathrm{tw}=8.02$ & 25 & $<0.0001^{* * * *}$ \\
\hline Urine toxicology (pos/neg) & $P: 12 / 14 \quad V: 13 / 13$ & $\mathrm{t} 1: 0 / 30 \quad \mathrm{t} 2: 0 / 30$ & - & - & - \\
\hline Hair toxicology $(\mathrm{pg} / \mathrm{mg})_{a}$ & $20377.80 \pm 23006.14$ & 0 & $\mathrm{tw}=4.25$ & 22 & $<0.0001$ \\
\hline \multicolumn{6}{|l|}{ Alcohol } \\
\hline Grams/week & $276.66 \pm 540.01$ & $58.54 \pm 52.59$ & $\mathrm{tw}=2.05$ & 25.4 & 0.051 \\
\hline \multicolumn{6}{|l|}{ Nicotine } \\
\hline Cigarettes/week & $95.54 \pm 68.67$ & $46.16 \pm 44.57$ & $\mathrm{tw}=3.14$ & 41.8 & $0.003^{\star *}$ \\
\hline \multicolumn{6}{|l|}{ Cannabis } \\
\hline Grams/week $^{\circ}$ & $2.42 \pm 6.42$ & $0.02 \pm 0.04$ & $\mathrm{tw}=1.88$ & 24 & 0.073 \\
\hline Urine toxicology (pos/neg) & $P: 6 / 20 \quad V: 7 / 19$ & $\mathrm{t} 1: 0 / 30 \quad \mathrm{t} 2: 0 / 30$ & - & - & - \\
\hline \multicolumn{6}{|l|}{ Amphetamine } \\
\hline Grams/week & $0.15 \pm 0.54$ & 0 & $\mathrm{tw}=1.38$ & 25 & 0.179 \\
\hline Urine toxicology (pos/neg) & $P: 1 / 25 \quad V: 0 / 26$ & $\mathrm{t} 1: 0 / 30 \quad \mathrm{t} 2: 0 / 30$ & - & - & - \\
\hline Hair toxicology amphetamine $(\mathrm{pg} / \mathrm{mg})_{\mathrm{b}}$ & $650.00 \pm 1149.13$ & 0 & $\mathrm{tw}=2.71$ & 22 & $0.013^{*}$ \\
\hline Hair toxicology methamphetamine $(\mathrm{pg} / \mathrm{mg})_{\mathrm{c}}$ & $392.39 \pm 1811.77$ & 0 & $\mathrm{tw}=1.04$ & 22 & 0.310 \\
\hline \multicolumn{6}{|l|}{ MDMA } \\
\hline Milligrams/week ${ }^{\circ}$ & $20.83 \pm 40.77$ & $0.64 \pm 2.87$ & $\mathrm{tw}=2.47$ & 24.2 & $0.021^{*}$ \\
\hline Hair toxicology MDMA $(\mathrm{pg} / \mathrm{mg}) \mathrm{d}$ & $1779.67 \pm 3164.51$ & $39.14 \pm 206.01$ & $\mathrm{tw}=2.63$ & 22.2 & $0.015^{\star}$ \\
\hline \multicolumn{6}{|l|}{ Opioid } \\
\hline Grams/week & $0.01 \pm 0.05$ & 0 & $\mathrm{tw}=1.43$ & 25 & 0.165 \\
\hline Urine toxicology (pos/neg) & $P: 0 / 26 \quad V: 0 / 26$ & $\mathrm{t} 1: 0 / 30 \quad \mathrm{t} 2: 0 / 30$ & - & - & - \\
\hline Hair toxicology morphine $(\mathrm{pg} / \mathrm{mg})$ & $120.00 \pm 84.85$ & 0 & $\mathrm{tw}=1.28$ & 22 & 0.213 \\
\hline \multicolumn{6}{|l|}{ GHB } \\
\hline Millilitres/week & $0.01 \pm 0.03$ & 0 & $t w=1$ & 25 & 0.327 \\
\hline \multicolumn{6}{|l|}{ Hallucinogens } \\
\hline Trips/week & $0.16 \pm 0.59$ & 0 & $\mathrm{tw}=1.34$ & 25 & 0.191 \\
\hline \multicolumn{6}{|l|}{ Ketamine } \\
\hline Grams/week & $0.02 \pm 0.06$ & 0 & $\mathrm{tw}=1.64$ & 25 & 0.113 \\
\hline Hair toxicology (pg/mg) & $756.63 \pm 2328.42$ & 0 & $\mathrm{tw}=1.56$ & 22 & 0.133 \\
\hline
\end{tabular}

Table S2. Means \pm standard deviations (s.d.) of substance use per group. Substance use in grams per week and frequency per week were based on self-report and averaged over the last six months. For self-report measures, sample size for healthy control individuals (HCI) and for cocaine-addicted individuals $(\mathrm{CAI})$, unless otherwise stated: $\mathrm{NCAI}=26 \mathrm{NHCI}=30$. Hair toxicology was analysed in two samples of total $6 \mathrm{~cm}$, if available. The average of the two samples captured substance use over the last six months. If the hair sample was shorter than 6 
$\mathrm{cm}$, only one sample for a shorter time period was analysed. Hair samples of four participants were missing due to boldness or dropout: $\mathrm{NCAI}=23 \mathrm{NHCI}=29$.

${ }^{\circ}$ Due to missing data in one self-report: $\mathrm{NCAI}=25 \mathrm{NHCI}=30$

a Sum of cocaine + cocaethylene + norcocaine, Cut-off value for cocaine $=500 \mathrm{pg} / \mathrm{mg}$

b Cut-off value for amphetamine $=200 \mathrm{pg} / \mathrm{mg}$

c Cut-off value for methamphetamine $=200 \mathrm{pg} / \mathrm{mg}$

d Cut-off value for MDMA $=200 \mathrm{pg} / \mathrm{mg}$

P: placebo, V: verum

MDMA: 3,4-methylenedioxymethamphetamine

GHB: gamma-Hydroxybutyric acid

$\mathrm{t}$ : independent $\mathrm{t}$-test for equal variances, 2-tailed

tw: Welch-test for unequal variances, 2-tailed

$* P<0.05, * * P<0.01$, *** $P<0.001, * * * * P<0.0001$ 
Impaired glutamate homeostasis...

\begin{tabular}{lccc}
\hline \multicolumn{4}{l}{ Table S3. Spectral quality over all groups and conditions } \\
\hline & Mean \pm s.d. & Minimum & Maximum \\
\hline SNR & $17.46 \pm 2.53$ & 11.00 & 24.00 \\
Linewidth (Hz) & $6.87 \pm 1.23$ & 4.34 & 11.75 \\
CRLBGlu (\%) & $5.07 \pm 1.24$ & 3.00 & 11.00 \\
\hline
\end{tabular}

Table S3. Data represent means \pm standard deviations (s.d.) of all proton magnetic resonance spectroscopy (1H-MRS) measurements (205) across conditions (neutral vs. cocaine film and placebo vs. $\mathrm{N}$-acetylcysteine) and groups (healthy control individuals vs. cocaine-addicted individuals). To describe the spectral quality assessed, we report signal-to-noise ratio (SNR) based on LCModel fittings, water linewidth (full-width at half-maximum peak height), and Cramér-Rao Lower Bounds (CRLBs) of the fit of glutamate. 
Impaired glutamate homeostasis...

\begin{tabular}{|c|c|c|c|c|c|}
\hline & $\begin{array}{l}\text { Cocaine-addicted } \\
\text { individuals }\end{array}$ & $\begin{array}{l}\text { Healthy control } \\
\text { individuals }\end{array}$ & & & \\
\hline & Mean \pm s.d. & Mean \pm s.d. & t-Value ${ }^{\circ}$ & $\mathrm{df}$ & $P$ Value \\
\hline \multicolumn{6}{|l|}{ Placebo } \\
\hline Neutral filma & $17.42 \pm 2.55$ & $17.39 \pm 2.62$ & -0.033 & 50 & 0.97 \\
\hline Cocaine filmb & $17.19 \pm 2.19$ & $17.31 \pm 2.75$ & 0.175 & 53 & 0.86 \\
\hline \multicolumn{6}{|l|}{ Verum } \\
\hline Neutral filmo & $18.13 \pm 2.80$ & $17.65 \pm 2.35$ & -0.648 & 47 & 0.52 \\
\hline Cocaine filmd & $17.59 \pm 2.61$ & $17.15 \pm 2.52$ & -0.601 & 47 & 0.55 \\
\hline
\end{tabular}

Table S4. Sample size N for cocaine-addicted individuals (CAI) and healthy control individuals (HCI) differed per condition:

a $\mathrm{NCAI}_{\mathrm{C}}=24 \mathrm{NHCI}=28$

$\mathrm{b}$ NCAI $=26 \mathrm{NHCI}_{\mathrm{H}}=29$

c NCAI $=23 \mathrm{NHCl}=26$

$\mathrm{d}$ NCAI $=22 \mathrm{NHCI}=27$

${ }^{\circ}$ Independent t-test, 2-tailed 
Impaired glutamate homeostasis...

\begin{tabular}{|c|c|c|c|c|c|}
\hline & $\begin{array}{l}\text { Cocaine-addicted } \\
\text { individuals }\end{array}$ & $\begin{array}{l}\text { Healthy control } \\
\text { individuals }\end{array}$ & & & \\
\hline & Mean \pm s.d. & Mean \pm s.d. & t-Value ${ }^{\circ}$ & $\mathrm{df}$ & $P$ Value \\
\hline \multicolumn{6}{|l|}{ Placebo } \\
\hline Neutral filma & $6.92 \pm 1.30$ & $6.75 \pm 1.33$ & -0.483 & 50 & 0.63 \\
\hline Cocaine filmb & $6.68 \pm 0.92$ & $6.85 \pm 1.14$ & 0.628 & 53 & 0.53 \\
\hline \multicolumn{6}{|l|}{ Verum } \\
\hline Neutral filmc & $6.67 \pm 1.14$ & $7.10 \pm 1.33$ & 1.219 & 47 & 0.23 \\
\hline Cocaine filmd & $6.92 \pm 1.28$ & $7.09 \pm 1.39$ & 0.455 & 47 & 0.65 \\
\hline
\end{tabular}

Table S5. Sample size N for cocaine-addicted individuals (CAI) and healthy control individuals (HCI) differed per condition:
a $\mathrm{NCAI}_{\mathrm{C}}=24 \mathrm{NHCI}=28$
b $\mathrm{NCAI}=26 \mathrm{NHCI}=29$
c $\mathrm{N}_{\mathrm{CAI}}=23 \mathrm{NHCI}=26$
$\mathrm{d} \mathrm{N}_{\mathrm{CAI}}=22 \mathrm{NHCI}=27$
${ }^{\circ}$ Independent t-test, 2-tailed 
Impaired glutamate homeostasis...

\begin{tabular}{|c|c|c|c|c|c|}
\hline & $\begin{array}{l}\text { Cocaine-addicted } \\
\text { individuals }\end{array}$ & $\begin{array}{l}\text { Healthy control } \\
\text { individuals }\end{array}$ & & & \\
\hline & Mean \pm s.d. & Mean \pm s.d. & t-Value ${ }^{\circ}$ & $\mathrm{df}$ & $P$ Value \\
\hline \multicolumn{6}{|l|}{ Placebo } \\
\hline Neutral filma & $5.00 \pm 1.10$ & $4.89 \pm 0.99$ & -0.368 & 50 & 0.71 \\
\hline Cocaine filmb & $4.92 \pm 1.16$ & $4.97 \pm 1.05$ & 0.142 & 53 & 0.89 \\
\hline \multicolumn{6}{|l|}{ Verum } \\
\hline Neutral filmc & $5.09 \pm 1.44$ & $5.35 \pm 1.79$ & 0.554 & 47 & 0.58 \\
\hline Cocaine filmd & $5.23 \pm 1.45$ & $5.19 \pm 0.83$ & -0.128 & 47 & 0.90 \\
\hline
\end{tabular}

Table S6. Sample size N for cocaine-addicted individuals (CAI) and healthy control individuals (HCI) differed per condition:
a $\mathrm{NCAI}_{\mathrm{C}}=24 \mathrm{NHCI}=28$
b $\mathrm{NCAI}=26 \mathrm{NHCI}=29$
c $\mathrm{N}_{\mathrm{CAI}}=23 \mathrm{NHCI}=26$
$\mathrm{d} \mathrm{N}_{\mathrm{CAI}}=22 \mathrm{NHCI}=27$
${ }^{\circ}$ Independent t-test, 2-tailed 
Impaired glutamate homeostasis...

\begin{tabular}{|c|c|c|c|c|c|}
\hline & $\begin{array}{l}\text { Cocaine-addicted } \\
\text { individuals }\end{array}$ & $\begin{array}{l}\text { Healthy control } \\
\text { individuals }\end{array}$ & & & \\
\hline & Mean \pm s.d. & Mean \pm s.d. & t-Value ${ }^{\circ}$ & $\mathrm{df}$ & $P$ Value \\
\hline \multicolumn{6}{|l|}{ Placebo } \\
\hline Neutral filma & $4.46 \pm 0.72$ & $4.64 \pm 0.91$ & 0.800 & 50 & 0.43 \\
\hline Cocaine filmb & $4.54 \pm 0.76$ & $4.66 \pm 0.97$ & 0.491 & 53 & 0.63 \\
\hline \multicolumn{6}{|l|}{ Verum } \\
\hline Neutral filmo & $4.35 \pm 0.65$ & $4.38 \pm 0.64$ & 0.200 & 47 & 0.84 \\
\hline Cocaine filmd & $4.59 \pm 0.85$ & $4.74 \pm 0.71$ & 0.670 & 47 & 0.51 \\
\hline
\end{tabular}

Table S7. Sample size N for cocaine-addicted individuals (CAI) and healthy control individuals (HCI) differed per condition:
a $\mathrm{N}_{\mathrm{CAI}}=24 \mathrm{NHCI}=28$
b $\mathrm{NCAI}_{\mathrm{C}}=26 \mathrm{NHCI}=29$
$\mathrm{c} \mathrm{N}_{\text {CAI }}=23 \mathrm{~N}$ HCI $=26$
$\mathrm{d}$ NCAI $=22 \mathrm{NHCI}=27$
${ }^{\circ}$ Independent t-test, 2-tailed 
Impaired glutamate homeostasis...

\begin{tabular}{|c|c|c|}
\hline \multirow[b]{3}{*}{ Fixed effects } & \multicolumn{2}{|c|}{ Dependent variable } \\
\hline & \multicolumn{2}{|c|}{ Glutamate + glutamine (GIx) } \\
\hline & estimate \pm s.e.m. & $P$ Value \\
\hline Intercept $(\mathrm{HCl})$ & $16.44 \pm 0.48$ & $<0.0001^{* \star *}$ \\
\hline $\mathrm{CAl}$ (versus $\mathrm{HCl}$ ) & $-1.37 \pm 0.70$ & 0.056 \\
\hline Cocaine film & $-1.28 \pm 0.52$ & $0.018^{*}$ \\
\hline CAI $\times$ cocaine film & $1.44 \pm 0.76$ & 0.066 \\
\hline Residual & 3.80 & \\
\hline Number of parameters & 5 & \\
\hline Number of participants & 56 & \\
\hline Number of data points & 107 & \\
\hline
\end{tabular}

Table S8. Estimates and standard errors (s.e.m.) for main effects (CAI, cocaine film) in reference to HCI as intercept, and interaction effect (CAI x cocaine film).

$\mathrm{CAI}=$ cocaine-addicted individuals

$\mathrm{HCI}=$ healthy control individuals

$* P<0.05, * * P<0.01, * * * P<0.001, * * * * P<0.0001$ 
Impaired glutamate homeostasis...

\begin{tabular}{lcc}
\hline Table S9. Random coefficient model for glutamate with N-acetylcysteine \\
\cline { 2 - 3 } & \multicolumn{2}{c}{ Dependent variable } \\
\cline { 2 - 3 } Fixed effects & \multicolumn{2}{c}{ Glutamate } \\
\hline Intercept (CAI) & $11.10 \pm 0.42$ & $P$ Value \\
$\mathrm{HCl}$ (versus CAI) & $1.48 \pm 0.58$ & $0.0001^{* * * *}$ \\
Cocaine film & $0.83 \pm 0.55$ & 0.14 \\
$\mathrm{HCl} \times$ cocaine film & $-1.65 \pm 0.75$ & $0.030^{*}$ \\
$\mathrm{~N}-\mathrm{AC}$ & $0.23 \pm 0.57$ & 0.69 \\
$\mathrm{HCl} \times \mathrm{N}-\mathrm{AC}$ & $-1.78 \pm 0.77$ & $0.024^{*}$ \\
Cocaine film $\times \mathrm{N}-\mathrm{AC}$ & $-1.16 \pm 0.80$ & 0.15 \\
$\mathrm{HCl} \times$ cocaine film $\times \mathrm{N}-\mathrm{AC}$ & $2.19 \pm 1.09$ & $0.047^{*}$ \\
Residual & 3.77 & \\
Number of parameters & 9 & \\
Number of participants & & \\
Number of data points & 56 & \\
\hline
\end{tabular}

Table S9. Estimates and standard errors (s.e.m.) for main effects (HCI, cocaine film, N-AC) in reference to CAI (intercept), and interaction effects.

$\mathrm{CAI}=$ cocaine-addicted individuals

$\mathrm{HCI}=$ healthy control individuals

$\mathrm{N}$-AC $=\mathrm{N}$-acetylcysteine

$* P<0.05, * * P<0.01, * * * P<0.001, * * * * P<0.0001$ 
Impaired glutamate homeostasis...

\begin{tabular}{cccc}
\multicolumn{2}{l}{ Table S10. Adverse events during $\mathrm{N}$-acetylcysteine or mannitol challenge } & \\
\hline Challenge & reaction & $\begin{array}{c}\text { Adverse events } \\
\text { severity }\end{array}$ & causality \\
\hline Mannitol & diarrhoea & mild & probable \\
& neck pain & moderate & unlikely \\
N-AC & nausea & mild & probable \\
& local allergic reaction & mild & unlikely \\
& herpes labialis & mild & unlikely \\
& transpiration & mild & possible \\
& Headache, vomitus & moderate & possible \\
\hline
\end{tabular}

Table S10. Overview of all adverse events that were reported during study participation with a two-day challenge of $2400 \mathrm{mg} /$ day $\mathrm{N}$-acetylcysteine (N-AC, verum) and of mannitol (placebo). Within the included sample, for intake of verum $\mathrm{N}=53$, for intake of placebo $\mathrm{N}=$ 56. Among all subjects, there were no serious adverse events observed, and severity of most events was rated as mild and two events as moderate. Causality between adverse events and the challenge were assessed either as certain, probable, possible or unlikely. 